\title{
Content Usage and the Most Frequently Read Articles of 2016
}

\author{
Phil Lupo, MLIS, Anne Victoria Neale, PhD, MPH, and \\ Marjorie A. Bowman, MD, MPA
}

2016 was the 29th year of print and 14th year of publication of the Journal of the American Board of Family Medicine (JABFM). Once again, we provide our annual report of articles published in 2016 that were of greatest interest to our readers. Table 1 shows the 3 most frequently read articles by issue in 2016. Once again, original research proves to be the most-read type of article.

In addition, we provide an update on the usage statistics of our content over the past 8 years. Figure 1 illustrates the number of total access events for FABFM content from 2009 to 2016. 2016 was the

Conflict of interest: The authors are editors and staff of the 7ABFM. busiest year for online usage activity in 7ABFM's history, with an average of 619,766 content usage requests a month, and a total of 7.43 million during the year. Content activity was up $16 \%$ in 2016 compared with content usage requests during 2015 and, when compared with 2009 , we note a $215 \%$ increase. Full-text articles were the most accessed during 2016, with an average of 223,311 monthly requests, followed by abstracts, with 191,517 average monthly requests, and then PDF articles, with 135,060 average monthly requests.

As always, we welcome reader feedback about published articles. Comments about specific articles can be posted to the $7 A B F M$ website (www. jabfm.org) by selecting "Responses" to the right of

Figure 1. Total access events for Journal of the American Board of Family Medicine content from 2009 to 2016. An access event occurs each time a user opens a new screen on the website.

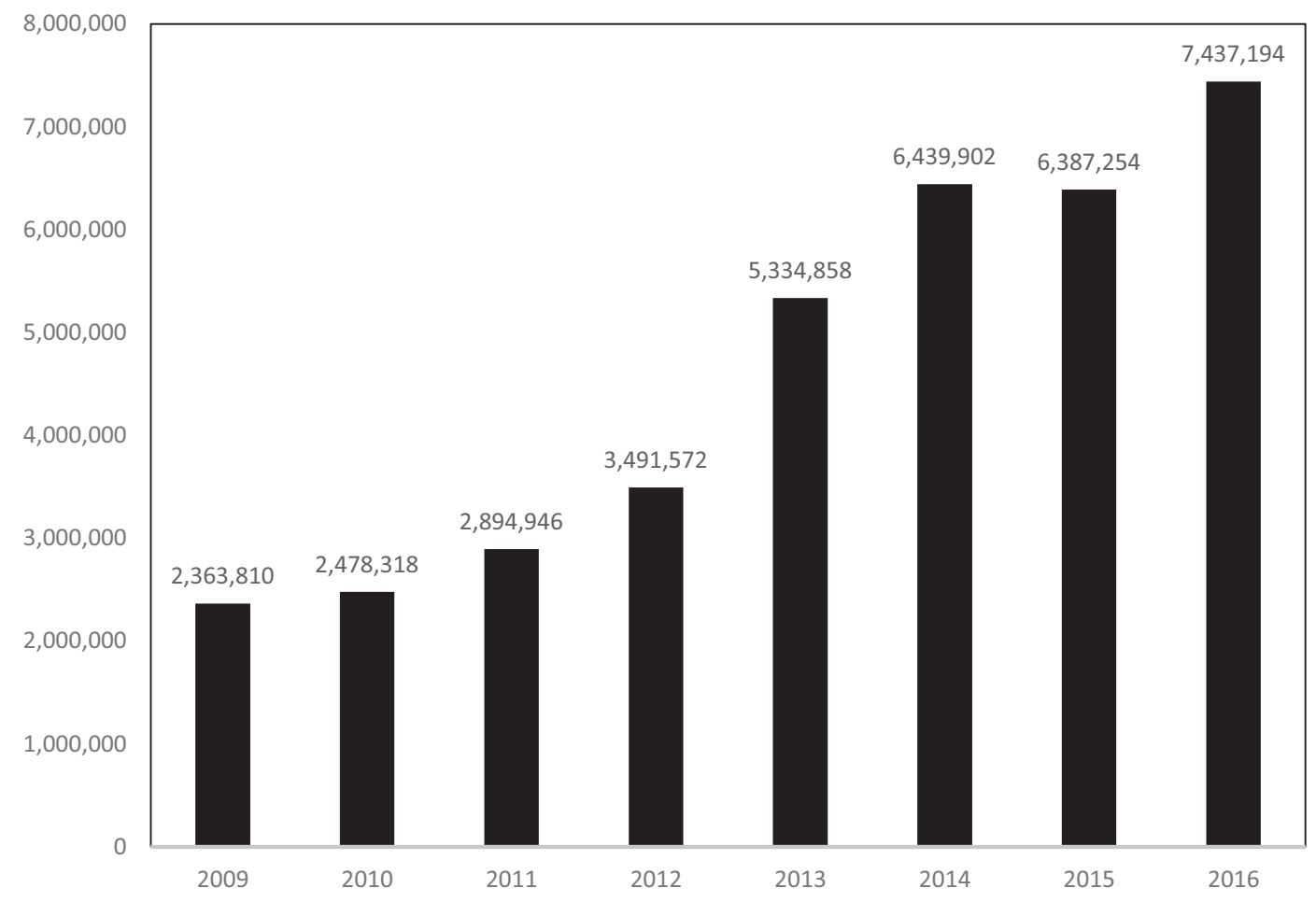


Table 1. The Three Most Frequently Read Articles in the Journal of the American Board of Family Medicine, 2016, Volume 29, By Issue

\begin{tabular}{|c|c|c|}
\hline Issue & Article & Article Type \\
\hline \multirow[t]{3}{*}{1 (January/February) } & $\begin{array}{l}\text { 1. Friedman A, Howard J, Shaw EK, Cohen DJ, Shahidi L, Ferrante JM. } \\
\text { Facilitators and barriers to care coordination in patient-centered } \\
\text { medical homes (PCMHs) from coordinators' perspectives }{ }^{1} \text {; } \\
\text { http://www.jabfm.org/content/29/1/90.full }\end{array}$ & Original research \\
\hline & $\begin{array}{l}\text { 2. Loskutova NY, Tsai AG, Fisher EB, et al. Patient navigators } \\
\text { connecting patients to community resources to improve diabetes } \\
\text { outcomes }{ }^{2} \text {; http://www.jabfm.org/content/29/1/78.full }\end{array}$ & Original research \\
\hline & $\begin{array}{l}\text { 3. Weiss BD, Brega AG, LeBlanc WG, et al. Improving the effectiveness } \\
\text { of medication review: guidance from the health literacy universal } \\
\text { precautions toolkit }{ }^{3} \text {; http://www.jabfm.org/content/29/1/18.full }\end{array}$ & Original research \\
\hline \multirow[t]{3}{*}{2 (March/April) } & $\begin{array}{l}\text { 1. Wolfe RM, Pomerantz J, Miller DE, Weiss-Coleman R, Solomonides } \\
\text { T. Obstructive sleep apnea: preoperative screening and postoperative } \\
\text { care }^{4} \text {; http://www.jabfm.org/content/29/2/263.full }\end{array}$ & Clinical review \\
\hline & $\begin{array}{l}\text { 2. Mainous AG III, Tanner RJ, Baker R. Prediabetes diagnosis and } \\
\text { treatment in primary care }{ }^{5} \text {; http://www.jabfm.org/content/ } \\
\text { 29/2/283.full }\end{array}$ & Brief report \\
\hline & $\begin{array}{l}\text { 3. Chien AL, Qi J, Rainer B, Sachs DL, Helfrich YR. Treatment of acne } \\
\text { in pregnancy } \text {; http://www.jabfm.org/content/29/2/254.full }\end{array}$ & Clinical review \\
\hline \multirow[t]{3}{*}{3 (May/June) } & $\begin{array}{l}\text { 1. Pinto AD, Glattstein-Young G, Mohamed A, Bloch G, Leung F-H, } \\
\text { Glazier RH. Building a foundation to reduce health inequities: routine } \\
\text { collection of sociodemographic data in primary care }{ }^{7} \text {; http://www. } \\
\text { jabfm.org/content/29/3/348.full }\end{array}$ & Original research \\
\hline & $\begin{array}{l}\text { 2. Lam CA, Sherbourne C, Tang L, et al. The impact of community } \\
\text { engagement on health, social, and utilization outcomes in depressed, } \\
\text { impoverished populations: secondary findings from a randomized } \\
\text { trial }^{8} \text {; http://www.jabfm.org/content/29/3/325.full }\end{array}$ & Original research \\
\hline & $\begin{array}{l}\text { 3. van Eeghen C, Littenberg B, Holman MD, Kessler R. Integrating } \\
\text { behavioral health in primary care using lean workflow analysis: a case } \\
\text { study }^{9} \text {; http://www.jabfm.org/content/29/3/385.full }\end{array}$ & Original research \\
\hline \multirow[t]{3}{*}{4 (July/August) } & $\begin{array}{l}\text { 1. Raffoul M, Moore M, Kamerow D, Bazemore A. A primary care panel } \\
\text { size of } 2500 \text { is neither accurate nor reasonable }{ }^{10} \text {; http://www.jabfm. } \\
\text { org/content/29/4/496.full }\end{array}$ & Special communication \\
\hline & $\begin{array}{l}\text { 2. Duane M, Contreras A, Jensen ET, White A. The performance of } \\
\text { fertility awareness-based method apps marketed to avoid pregnancy }{ }^{11} \text {; } \\
\text { http://www.jabfm.org/content/29/4/508.full }\end{array}$ & Brief report \\
\hline & $\begin{array}{l}\text { 3. DeFroda SF, Lamin E, Gil JA, Sindhu K, Ritterman S. Antibiotic } \\
\text { prophylaxis for patients with a history of total joint replacement }{ }^{12} \text {; } \\
\text { http://www.jabfm.org/content/29/4/500.full }\end{array}$ & Clinical review \\
\hline \multirow[t]{3}{*}{5 (September/October) } & $\begin{array}{l}\text { 1. Dexter EN, Fields S, Rdesinski RE, Sachdeva B, Yamashita D, Marino } \\
\text { M. Patient-provider communication: does electronic messaging reduce } \\
\text { incoming telephone calls? }{ }^{13} \text {; http://www.jabfm.org/content/ } \\
\text { 29/5/613.full }\end{array}$ & Original research \\
\hline & $\begin{array}{l}\text { 2. Litvin CB, Hyer JM, Ornstein SM. Use of clinical decision support to } \\
\text { improve primary care identification and management of chronic kidney } \\
\text { disease }(\mathrm{CKD})^{14} ; \text { http://www.jabfm.org/content/29/5/604.full }\end{array}$ & Original research \\
\hline & $\begin{array}{l}\text { 3. Cameron BJ, Bazemore AW, Morley CP. Lost in translation: NIH } \\
\text { funding for family medicine research remains limited }{ }^{15} \text {; http://www. } \\
\text { jabfm.org/content/29/5/528.full }\end{array}$ & Policy brief \\
\hline \multirow[t]{3}{*}{6 (November/December) } & $\begin{array}{l}\text { 1. Mainous AG III, Tanner RJ, Scuderi CB, Porter M, Carek PJ. } \\
\text { Prediabetes screening and treatment in diabetes prevention: the impact } \\
\text { of physician attitudes }{ }^{16} ; \text { http://www.jabfm.org/content } / 29 / 6 / 663 \text {.full }\end{array}$ & Original research \\
\hline & $\begin{array}{l}\text { 2. Shaughnessy AF, Cosgrove L, Lexchin JR. The need to systematically } \\
\text { evaluate clinical practice guidelines }{ }^{17} \text {; http://www.jabfm.org/content/ } \\
\text { 29/6/644.full }\end{array}$ & Commentary \\
\hline & $\begin{array}{l}\text { 3. Mospan GA, Wargo KA. 5-Day versus 10-Day course of } \\
\text { fluoroquinolones in outpatient males with a urinary tract infection } \\
\text { (UTI) }{ }^{18} \text {; http://www.jabfm.org/content/29/6/654.full }\end{array}$ & Original research \\
\hline
\end{tabular}

Source: Content usage statistics for the 7 ABFM, January 2016 through December 2016, HighWire Press. Accessed March 9, 2017. Rankings are updated monthly and based on hits received by articles archived on the FABFM website only. 
the article text. Letters to the editor can also be submitted for peer review and publication using the author portal.

To see this article online, please go to: http://jabfm.org/content/ 30/3/272.full.

\section{References}

1. Friedman A, Howard J, Shaw EK, Cohen DJ, Shahidi L, Ferrante JM. Facilitators and barriers to care coordination in patient-centered medical homes (PCMHs) from coordinators' perspectives. J Am Board Fam Med 2016;29:90-101.

2. Loskutova NY, Tsai AG, Fisher EB, et al. Patient navigators connecting patients to community resources to improve diabetes outcomes. J Am Board Fam Med 2016;29:78-89.

3. Weiss BD, Brega AG, LeBlanc WG, et al. Improving the effectiveness of medication review: guidance from the health literacy universal precautions toolkit. J Am Board Fam Med 2016;29:18-23.

4. Wolfe RM, Pomerantz J, Miller DE, Weiss-Coleman R, Solomonides T. Obstructive sleep apnea: preoperative screening and postoperative care. J Am Board Fam Med 2016;29:263-75.

5. Mainous AG III, Tanner RJ, Baker R. Prediabetes diagnosis and treatment in primary care. J Am Board Fam Med 2016;29:283-5.

6. Chien AL, Qi J, Rainer B, Sachs DL, Helfrich YR. Treatment of acne in pregnancy. J Am Board Fam Med 2016;29:254-62.

7. Pinto AD, Glattstein-Young G, Mohamed A, Bloch G, Leung F-H, Glazier RH. Building a foundation to reduce health inequities: routine collection of sociodemographic data in primary care. J Am Board Fam Med 2016;29:348-55.

8. Lam CA, Sherbourne C, Tang L, et al. The impact of community engagement on health, social, and utilization outcomes in depressed, impoverished populations: secondary findings from a randomized trial. J Am Board Fam Med 2016;29:325-38.
9. van Eeghen C, Littenberg B, Holman MD, Kessler R. Integrating behavioral health in primary care using lean workflow analysis: a case study. J Am Board Fam Med 2016;29:385-93.

10. Raffoul M, Moore M, Kamerow D, Bazemore A. A primary care panel size of 2500 is neither accurate nor reasonable. J Am Board Fam Med 2016;29: 496-9.

11. Duane M, Contreras A, Jensen ET, White A. The performance of fertility awareness-based method apps marketed to avoid pregnancy. J Am Board Fam Med 2016;29:508-11.

12. DeFroda SF, Lamin E, Gil JA, Sindhu K, Ritterman S. Antibiotic prophylaxis for patients with a history of total joint replacement. J Am Board Fam Med 2016;29:500-7.

13. Dexter EN, Fields S, Rdesinski RE, Sachdeva B, Yamashita D, Marino M. Patient-provider communication: does electronic messaging reduce incoming telephone calls? J Am Board Fam Med 2016;29: 613-9.

14. Litvin CB, Hyer JM, Ornstein SM. Use of clinical decision support to improve primary care identification and management of chronic kidney disease (CKD). J Am Board Fam Med 2016;29:604-12.

15. Cameron BJ, Bazemore AW, Morley CP. Lost in translation: NIH funding for family medicine research remains limited. J Am Board Fam Med 2016; 29:528-30.

16. Mainous AG III, Tanner RJ, Scuderi CB, Porter M, Carek PJ. Prediabetes screening and treatment in diabetes prevention: the impact of physician attitudes. J Am Board Fam Med 2016;29:663-71.

17. Shaughnessy AF, Cosgrove L, Lexchin JR. The need to systematically evaluate clinical practice guidelines. J Am Board Fam Med 2016;29:644-8.

18. Mospan GA, Wargo KA. 5-Day versus 10-Day course of fluoroquinolones in outpatient males with a urinary tract infection (U'TI). J Am Board Fam Med 2016;29:654-62. 